\title{
IS THE JONES POLYNOMIAL OF A KNOT REALLY A POLYNOMIAL?
}

\author{
STAVROS GAROUFALIDIS AND THANG TQ LÊ \\ Dedicated to Louis Kauffman on the occasion of his 60th birthday
}

\begin{abstract}
The Jones polynomial of a knot in 3-space is a Laurent polynomial in $q$, with integer coefficients. Many people have pondered why is this so, and what is a proper generalization of the Jones polynomial for knots in other closed 3-manifolds. Our paper centers around this question. After reviewing several existing definitions of the Jones polynomial, we show that the Jones polynomial is really an analytic function, in the sense of Habiro. Using this, we extend the holonomicity properties of the colored Jones function of a knot in 3-space to the case of a knot in an integer homology sphere, and we formulate an analogue of the AJ Conjecture. Our main tools are various integrality properties of topological quantum field theory invariants of links in 3-manifolds, manifested in Habiro's work on the colored Jones function.
\end{abstract}

\section{Contents}

1. Introduction 2

1.1. The Jones polvnomial of a knot 2

1.2. The Kauffman bracket of a knot 2

1.3. The quantum group approach 2

1.4. The TQFT and the perturbative approach 3

1.5. The Habiro ring 3

1.6. Statement of the results $\quad 4$

1.7. The colored Jones function is $q$-holonomid 4

1.8. The recurrence polvnomial 5

2. A review of Habiro's work $\quad 6$

2.1. The quantum group $U_{\alpha}\left(\mathfrak{s l}_{2}\right) \quad 6$

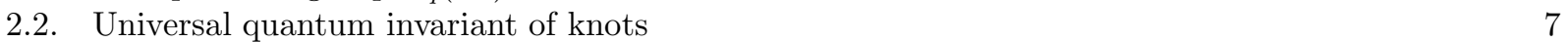

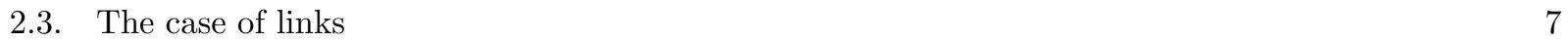

2.4. Invariants of integer homologv 3 -spheres $\quad 8$

2.5. Laplace transform $\quad 9$

3. A relative version $\quad 10$

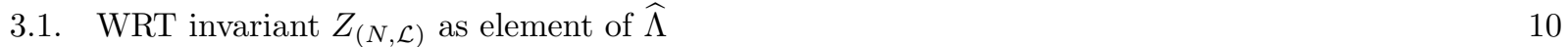

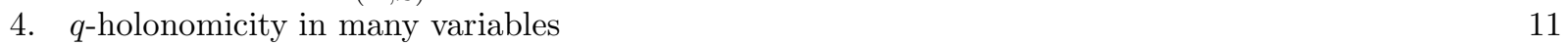

4.1. $q$-holonomicitv in manv variables $\quad 11$

4.2. Assembling $q$-holonomic functions 12

4.3. Examples of $q$-holonomic functions 12

4.4. $q$-holonomicitv of quantum invariants 12

$\begin{array}{ll}\text { References } & 13\end{array}$

Date: This edition: January 5, $2006 \quad$ First edition: January 5, 2006.

The authors were supported in part by NSF.

1991 Mathematics Classification. Primary 57N10. Secondary 57M25.

Key words and phrases: Knots, Jones polynomial, Kauffman bracket, Habiro ring, homology spheres, TQFT, holonomic functions, A-polynomial, AJ Conjecture. 


\section{Introduction}

1.1. The Jones polynomial of a knot. In 1985 Jones discovered the celebrated Jones polynomial of a knot/link in 3-space, see [J]. The framed version of the Jones polynomial of a framed oriented knot/link may be uniquely defined by the following skein theory:

$$
\left.q^{1 / 4} J_{Y}-q^{-1 / 4} J_{\curlywedge}=\left(q^{1 / 2}-q^{-1 / 2}\right) J\right), \quad J_{\text {unknot }}=q^{1 / 2}+q^{-1 / 2}
$$

From the above definition, the Jones polynomial $J_{L}$ of an oriented link $L$ lies in $\mathbb{Z}\left[q^{ \pm 1 / 4}\right]$.

An important and trivial observation is that knots may be considered to lie in other closed 3-manifolds other than $S^{3}$. The paper is centered around the following question:

Question 1. What is the Jones polynomial of a knot $K$ in a closed 3 -manifold $N$ ? In particular, is the Jones polynomial of a knot in a closed 3-manifold really a polynomial?

Before we reveal the answer, let us review some alternative definitions of the Jones polynomial, namely the Kauffman bracket approach, the quantum group approach, the TQFT (Witten-Reshetikhin-Turaev) approach, and the perturbative TQFT approach. Note that being polynomial is closely related to the integrality discussed in [Le3].

1.2. The Kauffman bracket of a knot. Soon after Jones's discovery, Kauffman gave a reformulation of the Jones polynomial in terms of the Kauffman bracket

$$
\langle\cdot\rangle \text { : Framed unoriented links in } S^{3} \longrightarrow \mathbb{Z}\left[A^{ \pm 1}\right]
$$

The Kauffman bracket is defined by the following skein theory (see [Ka]):

$$
\left.\left.\langle\rangle\rangle=A\langle\rangle+A^{-1}\right\rangle\right) \quad(\rangle, \quad\langle\bigcirc\rangle=-\left(A^{2}+A^{-2}\right)
$$

and the relation between the Kauffman bracket and the Jones polynomial is the following: if $L$ is an oriented $m$-component link projection with writhe $w(L)$, then

$$
\left.J_{L}\right|_{q^{1 / 4}=A}=(-1)^{m}\left(-A^{3}\right)^{-w(L)}\langle L\rangle
$$

Since the Kauffman bracket takes values in $\mathbb{Z}\left[A^{ \pm 1}\right]$, it implies once again that the Jones polynomial of an oriented knot/link in 3 -space takes values in $\mathbb{Z}\left[q^{ \pm 1 / 4}\right]$. Although the Kauffman bracket can be generalized to framed unoriented links in a closed 3-manifold, the resulting Kauffman bracket skein module is an interesting and not well-understood object. Most important, the Kauffman bracket skein module of a closed 3-manifold is not a ring, but rather a module over the ring $\mathbb{Z}\left[A^{ \pm 1}\right]$.

1.3. The quantum group approach. Using quantum group technology (see $\mathrm{RT}$, Tu] ), one may define the colored Jones polynomial of a framed oriented knot in 3-space. Let us postpone the technical details to a later Section 2.1 and concentrate on a main idea: the notion of color.

Suppose $L$ is a framed $r$-component link in $S^{3}$ with ordered components. The Jones polynomial of $L$ is a powerful invariant that takes values in the Laurent polynomial ring

$$
\mathcal{R}:=\mathbb{Z}\left[q^{ \pm 1 / 4}\right]
$$

An even more powerful invariant is the colored Jones function

$$
J_{L}: \mathbb{N}^{r} \longrightarrow \mathcal{R}
$$

which encodes the Jones polynomial of $L$ together with its parallels. Here $\mathbb{N}$ is the set of positive integers, and $J_{L}\left(n_{1}, \ldots, n_{r}\right)$ is the quantum $\mathfrak{s l}_{2}$ invariant of the link $L$ with colors the irreducible $\mathfrak{s l}_{2}$-modules of dimensions $n_{1}, \ldots, n_{r}$. When $n_{1}=\cdots=n_{r}=2$, the polynomial $J_{L}\left(n_{1}, \ldots, n_{r}\right)$ is the Jones polynomial. Here we use the normalization such that when $U$ is the unknot of 0 framing,

$$
J_{U}(n)=[n]:=\frac{q^{n / 2}-q^{-n / 2}}{q^{1 / 2}-q^{-1 / 2}} .
$$


1.4. The TQFT and the perturbative approach. Let us fix a framed oriented knot $K$ in a 3-manifold $N$ and a nonnegative integer $n \in \mathbb{N}$. The Witten-Reshetikhin-Turaev (WRT) invariant $Z_{(N, K), \xi, V_{n}} \in \mathbb{Q}(\xi)$ is a generalization of the colored Jones polynomial and can be defined under the framework of topological quantum field theory (TQFT in short); see [Tu. Here $V_{n}$ is the $n$-dimensional irreducible representation of $\mathfrak{s l}_{2}$ and $\xi$ is a complex root of unity of order divisible by 4 . When $M=S^{3}$, and with the proper normalization, we may identify the WRT invariant with the colored Jones polynomial as follows:

$$
Z_{\left(S^{3}, K\right), \xi, V_{n}}=\operatorname{ev}_{\xi} J_{K}(n),
$$

where

$$
\mathrm{ev}_{\xi}: \mathbb{Z}\left[q^{ \pm 1 / 4}\right] \longrightarrow \mathbb{Z}[\xi]
$$

is the evaluation $q^{1 / 4}=\xi$. From the point of view of TQFT, it is not clear why the Jones polynomial is even a polynomial.

Perturbative quantum field theory also constructs an invariant $Z_{(N, K), V_{n}}^{\text {pert }}$ which takes values in the power series ring $\mathbb{Q}[[h]]$. When $M=S^{3}, Z_{\left(S^{3}, K\right), V_{n}}^{\text {pert }}$ is the composition of the Kontsevich integral of a knot with the $\mathfrak{s l}_{2}$ weight system, and when $N$ is arbitrary, $Z_{(N, K), V_{n}}^{\text {pert }}$ is the composition of the LMO invariant with the $\mathfrak{s l}_{2}$ weight system, see [LMO and $\left[\mathrm{A}\right.$. When $M=S^{3}$, the main identity is:

$$
\operatorname{ev}_{h} Z_{K}(n)=Z_{(N, K), V_{n}}^{\text {pert }} \in \mathbb{Q}[[h]]
$$

where

$$
\mathrm{ev}_{h}: \mathbb{Z}\left[q^{ \pm 1 / 4}\right] \longrightarrow \mathbb{Q}[[h]]
$$

is the evaluation $q=e^{h}$; see for example $\underline{\text { O] }}$. Thus, from the point of view of perturbative TQFT the Jones polynomial is a formal power series in $h$.

1.5. The Habiro ring. Despite the apparent failure of TQFT to explain the polynomial aspect of the Jones polynomial, there is one gain. Namely let us fix $(N, K)$ a natural number $n$ and let $\Omega_{4}$ denote the set of complex roots of unity whose order is divisible by 4 . Then, the WRT invariant gives a function:

$$
Z_{(N, K), V_{n}}: \Omega_{4} \longrightarrow \mathbb{C} \text {. }
$$

This function is not continuous, and does not have nice analytic properties. Habiro introduced an alternative notion of analytic functions. The latter are by definition elements of the Habiro ring, defined by:

$$
\widehat{\mathbb{Z}[q]}:=\lim _{\leftarrow n} \mathbb{Z}[q] /\left((1-q)\left(1-q^{2}\right) \ldots\left(1-q^{n}\right)\right) .
$$

The ring $\widehat{\mathbb{Z}[q]}$ can be considered as the set of all series of the form

$$
f(q)=\sum_{n=0}^{\infty} f_{n}(q)(1-q)\left(1-q^{2}\right) \ldots\left(1-q^{n}\right), \quad \text { where } \quad f_{n}(q) \in \mathbb{Z}[q],
$$

with the warning that $f(q)$ does not uniquely determine $\left(f_{n}(q)\right)$.

It turns out that the Habiro ring $\widehat{\mathbb{Z}[q]}$ shares many properties with the ring of germs of complex analytic functions, (see [Ha1]) and plays an important role in Quantum Topology. In particular, elements of the Habiro ring

(a) can be differentiated with respect to $q$,

(b) can be evaluated at the set $\Omega$ of complex roots of unity,

(c) have Taylor series expansions that uniquely determine them,

(d) form an integral domain.

These properties suggest that we consider $\widehat{\mathbb{Z}[q]}$ as a class of "analytic functions" with domain $\Omega$. For proofs of these properties, we refer the reader to $\underline{\mathrm{Ha} 2}$.

Let us comment a bit further on these properties. (a) is obvious from Equation (4). (b) also follows from (4) because when $q$ is a root of unity, only a finite number of terms in the right hand side of Equation (4) are not 0 , hence $f(q)$ is defined as a complex number. Thus one can consider every $f \in \widehat{\mathbb{Z}[q]}$ as a function 
with domain $\Omega$ the set of roots of unity. (a) and (b) imply that elements of the Habiro ring have Taylor series expansions at every complex root of unity. In particular, every $f \in \widehat{\mathbb{Z}[q]}$ has a Taylor expansion

$$
T_{1}(f) \in \mathbb{Z}[[q-1]] \subset \mathbb{Q}[[h]]
$$

(where $q=e^{h}$ ). What is nontrivial is the fact that $T_{1}(f)$ uniquely uniquely determines $f$. (d) follows immediately from (c). Another nontrivial fact is that if $f(\xi)=g(\xi)$ at infinitely many roots $\xi$ of prime power orders, then $f=g$ in $\widehat{\mathbb{Z}[q]}$. Therefore, one has the following corollary of Habiro's results:

Proposition 1.1. The map $\widehat{\mathbb{Z}[q]} \rightarrow \prod_{n=1}^{\infty} \mathbb{Z}\left[\xi_{n}\right], f \rightarrow\left(f\left(\xi_{1}\right), f\left(\xi_{2}\right), \ldots\right)$ is injective. Here $\xi_{n}=\exp (2 \pi i / n)$.

An example of a non-trivial element of the Habiro ring (of interest to quantum topology) is the following element:

$$
f(q)=\sum_{n=0}^{\infty}(1-q)\left(1-q^{2}\right) \ldots\left(1-q^{n}\right)
$$

studied by Kontsevich (unpublished), Zagier and the second author; see [Za] and [Le3].

1.6. Statement of the results. Ending our discussion on the Habiro ring, let us get back to Equation (2), and let us assume for simplicity that $K$ is a 0 -framed knot in an integer homology 3 -sphere $N$. It turns out that there is a function $f \in q^{n / 2} \widehat{\mathbb{Z}[q]}$ such that for every $\xi \in \Omega_{4}$,

$$
Z_{(N, K), V_{n}, \xi}=\mathrm{ev}_{\xi} f
$$

Proposition 1.1 shows that this $f$ is an invariant of the triple $N, K, n$, and we denote it by $J_{(N, K)}(n)$. Note that if $n$ is odd, then $J_{(N, K)}(n) \in \widehat{\mathbb{Z}[q]}$, otherwise $J_{(N, K)}(n) \in q^{1 / 2} \widehat{\mathbb{Z}[q]}$. Our next definition answers Question 1 and also explains the title of the paper:

Definition 1.2. The colored Jones function $J_{(N, K)}$ of a 0 -framed knot $K$ in an integer homology 3 -sphere $N$ is defined by:

$$
J_{(N, K)}: \mathbb{N} \longrightarrow \widehat{\Lambda}
$$

where

$$
\begin{aligned}
\widehat{\Lambda} & :=\widehat{\mathbb{Z}[q]}+q^{1 / 2} \widehat{\mathbb{Z}[q]} \\
& \cong \widehat{\mathbb{Z}[q]}[x] /\left(x^{2}-q\right) .
\end{aligned}
$$

Remark 1.3. If $K$ is an 0-framed knot in an integer homology 3-sphere $N$ and $K_{f}$ denotes the knot $K$ with framing $f$ (where $f \in \mathbb{Z}$ ), then

$$
J_{\left(N, K_{f}\right)}=q^{\frac{n^{2}-1}{4}} J_{(N, K)} .
$$

Therefore, the colored Jones function of a framed knot in an integer homology 3-sphere takes value in the ring

$$
\widehat{\mathbb{Z}[q]}[x] /\left(x^{4}-q\right) .
$$

1.7. The colored Jones function is $q$-holonomic. Now that we know what the Jones polynomial of a knot in an integer homology 3-sphere really is, we may extend known results and conjectures of the Jones polynomial of a knot in $S^{3}$ to knots in integer homology 3-spheres. One of these results, due to the authors, is the fact that the colored Jones function of a knot in $S^{3}$ is $q$-holonomic, see GL. Let us recall this notion in our setting.

Define the linear operators $L$ and $M$ acting on maps $f$ from $\mathbb{N}$ to an $\mathbb{Z}\left[q^{1 / 2}\right]$-module by:

$$
(M f)(n)=q^{n / 2} f(n), \quad(L f)(n)=f(n+1) .
$$

It is easy to see that $L M=q^{1 / 2} M L$, and that $L, M$ generate the quantum plane $\mathcal{A}$, the non-commutative ring with presentation

$$
\mathcal{A}=\mathbb{Z}\left[q^{ \pm 1 / 2}\right]\langle M, L\rangle /\left(L M=q^{1 / 2} M L\right) .
$$


The recurrence ideal of the discrete function $f$ is the left ideal $I_{f}$ in $\mathcal{A}$ that annihilates $f$ :

$$
I_{f}=\{P \in \mathcal{A} \mid \quad P f=0\} .
$$

We say that $f$ is $q$-holonomic, or $f$ satisfies a linear recurrence relation, if $I_{f} \neq 0$. In GL we proved that for every knot $K$ in $S^{3}$, the function $J_{K}$ is $q$-holonomic. In other words, $J_{K}$ satisfies a linear recursion relation with coefficients Laurent polynomials in $q^{1 / 2}$ and $q^{n / 2}$.

Theorem 1. For every 0-framed knot $K$ in an integer homology 3-sphere $N$, the colored Jones function $J_{(N, K)}$ is q-holonomic.

The main ideas behind the proof of the above theorem is that

- every pair $(N, K)$ as above is obtained from unit-framed surgery from an algebraically split link $K \cup L$ in $S^{3}$.

- The function $J_{(N, K)}$ is obtained from the colored Jones function $J_{K \cup L}$ by elimination of the variables corresponding to $L$.

- The function $J_{K \cup L}$ is $q$-holonomic in all its variables (see section 4.4 below), by GL].

- Elimination preserves $q$-holonomicity.

1.8. The recurrence polynomial. Let $I_{(N, K)}$ denote the recurrence ideal of $J_{(N, K)}$. Then $I_{(N, K)}$ is a left ideal of $\mathcal{A}$, which is not a principal ideal domain. Hence $I_{(N, K)}$ might not be generated by a single element. The first author $\mathrm{Ga}$ noticed that by adding to $\mathcal{A}$ all the inverses of polynomials in $M$ one gets a principal ideal domain $\tilde{\mathcal{A}}$, and hence from the ideal $I_{(N, K)}$ one can define a polynomial invariant. Formally, let $\mathbb{Q}\left(q^{1 / 2}, M\right)$ be the fractional field of the polynomial ring $\mathcal{R}[M]$. Let $\tilde{\mathcal{A}}$ be the set of all polynomials in the variable $L$ with coefficients in $\mathbb{Q}\left(q^{1 / 2}, M\right)$ :

$$
\tilde{\mathcal{A}}=\left\{\sum_{k=0}^{\infty} a_{k}(M) L^{k} \mid \quad a_{k}(M) \in \mathbb{Q}\left(q^{1 / 2}, M\right), a_{k}=0 \quad k \gg 0\right\}
$$

with multiplication given by

$$
a(M) L^{k} \cdot b(M) L^{l}=a(M) b\left(q^{k / 2} M\right) L^{k+l} .
$$

It is known that $\tilde{\mathcal{A}}$ is a twisted polynomial ring (an Ore extension of $\mathbb{Q}\left(q^{1 / 2}, M\right)$ ), and consequently $\tilde{\mathcal{A}}$ is a principal left-ideal domain, and $\mathcal{A}$ embeds as a subring of $\tilde{\mathcal{A}}$. The ideal extension $\tilde{I}_{(N, K)}:=\tilde{\mathcal{A}} I_{(N, K)}$ is then generated by a single polynomial

$$
\alpha_{(N, K)}(L, M, q)=\sum_{i=0}^{n} \alpha_{(N, K), i}(M, q) L^{i}
$$

where the degree in $L$ is assumed to be minimal and all the coefficients $\alpha_{(N, K), i}(M, q) \in \mathbb{Z}\left[q^{ \pm 1 / 2}, M\right]$ are assumed to be co-prime. That $\alpha_{(N, K)}$ can be chosen to have integer coefficients follows from the fact that $J_{(N, K)}(n) \in \widehat{\Lambda}$. It is clear that $\alpha_{(N, K)}(L, M, q)$ annihilates $J_{(N, K)}$, and hence it is in the recurrence ideal $I_{(N, K)}$. Note that $\alpha_{(N, K)}(M, L ; q)$ is defined up to a factor $\pm q^{a / 2} M^{b}, a, b \in \mathbb{Z}$. We will call $\alpha_{(N, K)}$ the recurrence polynomial of $(N, K)$.

Let us say that two non-zero polynomials $p_{1}$ and $p_{2}$ in variables $L$ and $M$ are $M$-essentially equal (and write $p_{1} \stackrel{M}{=} p_{2}$ ) if their ratio is a function of $M$ alone. The next conjecture generalizes the AJ Conjecture of the first author (see $\mathrm{Ga}]$ ):

Conjecture 1. For every knot $K$ in an integer homology 3-sphere $N$, we have:

$$
\alpha_{(N, K)}(L, M, 1) \stackrel{M}{=} A_{(N, K)}(L, M),
$$

where $A_{(N, K)}(L, M)$ is the A-polynomial of $(N, K)$ defined by CCGLS.

For some partial results confirming the conjecture, see [Hil Le2, Ga]. In particular, in Le2 the second author used Kauffman bracket modules to established the conjecture for a large class of 2-bridge knots in $S^{3}$. The above conjecture compares a quantum invariant (the recurrence ideal of the colored Jones function) with 
a classical one (namely the $A$-polynomial). One motivation of the conjecture is the dream of quantization and semiclassical analysis, in the context of 3-manifolds with torus boundary. Another motivation is the fact that the Kauffman bracket skein module is in a sense a quantization of the coordinate ring of the $\mathrm{SL}_{2}(\mathbb{C})$ character variety of a 3 -manifold; see $[\mathrm{PS}]$. The $A$-polynomial is the coordinate ring of the $\mathrm{SL}_{2}(\mathbb{C})$ character variety of the knot complement, viewed from the boundary torus. On the other hand, the recurrence polynomial is in a sense a quantization of the classical coordinate ring. Thus, we are back to the Kauffman bracket skein module. And with this happy thought in mind, we end this section.

\section{A REVIEW OF HABIRO'S WORK}

In this section we review Habiro's work on the integrality of the colored Jones polynomial and the WRT invariants of links in integer homology 3-spheres. For a detailed discussion, we refer the reader to Ha1 and Ha3.

2.1. The quantum group $U_{q}\left(\mathfrak{s l}_{2}\right)$. We begin by reviewing some necessary representation theory of quantum groups.

We will use the following analogs of quantum integers and quantum factorials:

$$
\{n\}=q^{n / 2}-q^{-n / 2}, \quad\{n\} !=\prod_{i=1}^{n}\{i\} !
$$

Let

$$
C(n, k):=\prod_{j=n-k}^{n+k}\{j\}=\frac{\{n+k\} !}{\{n-k-1\} !}
$$

Notice that $C(n, k)=0$ for $k>n$. Also $C(n, k) \in q^{n / 2} \mathbb{Z}\left[q^{ \pm 1}\right]$ and hence $\{n\} C(n, k) \in \mathbb{Z}\left[q^{ \pm 1}\right]$.

Consider the quantized enveloping algebra $U_{q}\left(\mathfrak{s l}_{2}\right)$ be defined as Jantzen's book Jan, except that our $q$ is equal to $q^{2}$ of [Jan], and our ground field is extended to $\mathbb{C}\left(q^{1 / 4}\right)$, instead of $\mathbb{C}\left(q^{1 / 2}\right)$ as in $[\mathrm{Jan}$.

The theory of type $1 U_{q}\left(\mathfrak{s l}_{2}\right)$-modules is totally similar to that of $\mathfrak{s l}_{2}$. For each positive integer there is a unique irreducible type $1 U_{q}\left(\mathfrak{s l}_{2}\right)$-module $V_{n}$, see eg Jan (where $V_{n}$ is denoted by $L(n,+)$ ). Let $\mathbf{R}$ be the Grothendieck ring of finite-dimensional type $1 U_{q}\left(\mathfrak{s l}_{2}\right)$-modules, tensored by $\mathbb{C}\left(q^{1 / 4}\right)$. Then $\mathbf{R}$ is freely spanned by $V_{1}, V_{2}, \ldots$, and as an algebra is isomorphic to the polynomial algebra $\mathbb{C}\left(q^{1 / 4}\right)\left[V_{2}\right]$. Let $\mathbf{R}^{e}$ denote the subspace of $\mathbf{R}$ spanned by even powers of $V_{2}$. For every framed, $r$-component, oriented link $\mathcal{L}$ whose components are ordered, the quantum invariant $J_{\mathcal{L}}$, extended linearly, can be considered as a function from $\mathbf{R}^{r}$ to $\mathbb{C}\left(q^{1 / 4}\right)$.

There is a symmetric bilinear form on $\mathbf{R}$ :

$$
\mathbf{R} \times \mathbf{R} \longrightarrow \mathbb{C}\left(q^{1 / 4}\right), \quad\langle V, U\rangle:=U^{V}
$$

Here the right hand side is the colored Jones polynomial of the Hopf link, colored by $V$ and $U$. For example, one has $\left\langle V_{n}, V_{m}\right\rangle=[n m]$.

Habiro defines the following elements in $\mathbf{R}$ :

$$
\begin{gathered}
S_{k}:=\prod_{i=1}^{k}\left(V_{2}^{2}-\{i\}^{2}\right), \quad \text { and } \\
P_{k}:=\prod_{i=1}^{k}\left(V_{2}-q^{(2 i-1) / 2}-q^{-(2 i-1) / 2}\right), \quad P_{k}^{\prime}:=\frac{P_{k}}{\{k\} !}, \quad P_{k}^{\prime \prime}:=\frac{\{1\}}{\{2 k+1\} !} P_{k} .
\end{gathered}
$$

It is clear that $\left\{S_{k}\right\}_{k \in \mathbb{N}}$ and $\left\{P_{k}\right\}_{k \in \mathbb{N}}$ are bases of $\mathbf{R}^{e}$ and $\mathbf{R}$ respectively. Moreover, the two bases are dual under the pairing (8). That is, we have:

$$
\left\langle P_{k}^{\prime \prime}, S_{n}\right\rangle=\delta_{n, k}
$$


It's easy to show that

$$
\left\langle V_{n}, S_{k}\right\rangle=\frac{C(n, k)}{\{1\}} .
$$

2.2. Universal quantum invariant of knots. Suppose $K$ is a 0 -framed oriented knot in $S^{3}$. Then $J_{K}$ can be considered as a $\mathbb{C}\left(q^{1 / 4}\right)$-linear map from $\mathbf{R}$ to the ground field $\mathbb{C}\left(q^{1 / 4}\right)$. Using the bilinear form (8) one can also consider each $S_{k}$ as a linear form on $\mathbf{R}$. Habiro showed that $J_{K}$ is always an infinite linear combination of the $S_{k}$, i.e. for every $V \in \mathbf{R}$,

$$
J_{K}(V)=\sum_{k=0}^{\infty} C_{K}(k)\left\langle V, S_{k}\right\rangle
$$

It is easy to show that for every $V \in \mathbf{R}$, if $k$ is big enough then $\left\langle V, S_{k}\right\rangle=0$, hence the right hand side has a meaning for every $V \in \mathbf{R}$. Using the orthogonality (9) one see that

$$
C_{K}(k)=J_{K}\left(P_{k}^{\prime \prime}\right)
$$

A priori $C(k)$ belongs to the ground field $\mathbb{C}\left(q^{1 / 4}\right)$. A difficult result of Habiro is that $C(k)$ is always a Laurent polynomial in $q$ with integer coefficients, $C(k) \in \mathbb{Z}\left[q^{ \pm 1}\right]$.

Using Equation (11) for $V=V_{n}$ and (10), one has

$$
J_{K}(n)=\sum_{k=0}^{\infty} C_{K}(k) C(n, k) /\{1\} .
$$

2.3. The case of links. Suppose $\mathcal{L}$ is an algebraically split $r$-component link in $S^{3}$ with 0 framing on each component. Then it is known that $J_{\mathcal{L}}\left(n_{1}, \ldots, n_{r}\right)$ is in $\mathbb{Z}\left[q^{ \pm 1}\right]$ or in $q^{1 / 2} \mathbb{Z}\left[q^{ \pm 1}\right]$, according as $n_{1}+\cdots+n_{r}-r$ is even or odd.

Equation (11) can be generalized to the link case, and we have the fundamental equation:

$$
J_{\mathcal{L}}\left(W_{1}, \ldots, W_{r}\right)=\sum_{k_{i}=0}^{\infty} C_{\mathcal{L}}\left(k_{1}, \ldots, k_{r}\right)\left\langle W_{1}, S_{k_{1}}\right\rangle \ldots\left\langle W_{r}, S_{k_{r}}\right\rangle,
$$

for every $W_{1}, \ldots, \mathcal{W}_{r} \in \mathbf{R}$, and

The analogue of Equation (12) is:

$$
J_{\mathcal{L}}\left(n_{1}, \ldots, n_{r}\right)=\sum_{k_{1}, \ldots, k_{r}=0}^{\infty} C_{\mathcal{L}}\left(k_{1}, \ldots, k_{r}\right) \prod_{i=1}^{r} \frac{C\left(n_{i}, k_{i}\right)}{\{1\}} .
$$

Unlike the knot case, the coefficient $C_{\mathcal{L}}\left(k_{1}, \ldots, k_{r}\right)$ of the right hand side is not always a Laurent polynomial, but rather a rational function in $q$. One of the main results of Habiro is the integrality of a minor modification $\widetilde{C}_{L}$ of the cyclotomic function $C_{L}$. Let us define

$$
\begin{aligned}
\widetilde{C}_{\mathcal{L}}\left(k_{1}, \ldots, k_{r}\right) & :=J_{\mathcal{L}}\left(P_{k_{1}}^{\prime}, \ldots, P_{k_{r}}^{\prime}\right) \\
& =C_{\mathcal{L}}\left(k_{1}, \ldots, k_{r}\right) \frac{\left\{2 k_{1}+1\right\} !}{\left\{k_{1}\right\} !\{1\}} \ldots \frac{\left\{2 k_{r}+1\right\} !}{\left\{k_{r}\right\} !\{1\}}
\end{aligned}
$$

Habiro proves that

Theorem 2. Ha1, Thm.3.3] If $L$ is algebraically split and zero framed link in $S^{3}$, then

$$
\widetilde{C}_{L}\left(k_{1}, \ldots, k_{r}\right) \in \frac{\{2 m+1\} !}{\{m\} !\{1\}} \mathbb{Z}\left[q^{ \pm 1 / 2}\right],
$$

where $m=\max \left\{k_{1}, \ldots, k_{r}\right\}$. 
The important thing is that for every $n, \frac{\{2 n+1\} !}{\{n\} !\{1\}}$ is divisible by $(1-q) \ldots\left(1-q^{n}\right)$. This guarantees that for any sequence $f(k) \in \mathbb{Z}\left[q^{ \pm 1}\right]$ (for $k \in \mathbb{N}$ ), the series

$$
\sum_{k=0}^{\infty} f(k) \frac{\{2 k+1\} !}{\{k\} !\{1\}}
$$

converges in $\widehat{\Lambda}$.

2.4. Invariants of integer homology 3 -spheres. Suppose $N$ is an integer homology 3 -sphere, which is obtained by surgery on $S^{3}$ along an algebraically split $r$-component link $\mathcal{L}$, with framing $f_{1}= \pm 1, \ldots, f_{r}=$ \pm 1 . Let $\mathcal{L}^{(0)}$ be the link $\mathcal{L}$ with all framing switched to 0 .

Habiro introduced the following elements $\omega^{ \pm 1}$ in some completion of $\mathbf{R}$ :

$$
\begin{aligned}
\omega & =\sum_{j=0}^{\infty} q^{j(j+3) / 4} P_{j}^{\prime} \\
\omega^{-1} & =\sum_{j=0}^{\infty}(-1)^{j} q^{-j(j+3) / 4} P_{j}^{\prime}
\end{aligned}
$$

Although each of $w^{ \pm 1}$ is an infinite sum of elements in $\mathbf{R}$, Theorem 2 (with the remark after the theorem) shows that $J_{\mathcal{L}^{(0)}}\left(w^{ \pm 1}, \ldots, w^{ \pm 1}\right)$ always belongs to $\widehat{\mathbb{Z}[q]}$.

Using a version of Kirby's calculus for algebraically split links, Habiro proved that

$$
J_{N}:=J_{\mathcal{L}^{(0)}}\left(w^{-f_{1}}, \ldots, w^{-f_{r}}\right) \in \widehat{\mathbb{Z}[q]}
$$

is an invariant of the integer homology 3 -sphere $N$, i.e. does not depend on the choice of the surgery link $\mathcal{L}$. Moreover, one has the following

Proposition 2.1. (Habiro) The evaluation of $J_{N}$ (as an element of $\widehat{\mathbb{Z}[q]}$ ) at a root of unity coincides with the WRT invariant $Z_{N}$ at that same root of unity.

Proof. We give a proof for this fact here, since we will adapt the proof for the relative case later.

Orthogonality (that is Equations (9) and (10)) implies that

$$
\left\langle P_{k}^{\prime}, S_{n}\right\rangle=\delta_{n, k} \frac{\{2 k+1\} !}{\{k\} !\{1\}}
$$

Thus, for $f= \pm 1$ we have:

$$
\left\langle\omega^{-f}, S_{k}\right\rangle=\left\langle(-f q)^{-f k(k+3) / 4} P_{k}^{\prime}, S_{k}\right\rangle=(-f q)^{-f k(k+3) / 4} \frac{\{2 k+1\} !}{\{k\} !\{1\}}
$$

This, together with Equation (13) implies that

$$
\begin{aligned}
J_{N} & =\sum_{k_{1}, \ldots, k_{r}=0}^{\infty} C\left(k_{1}, \ldots, k_{r}\right)\left\langle\omega^{-f_{1}}, S_{k_{1}}\right\rangle \ldots\left\langle\omega^{-f_{r}}, S_{k_{r}}\right\rangle \\
& =\sum_{k_{1}, \ldots, k_{r}=0}^{\infty} C\left(k_{1}, \ldots, k_{r}\right) \prod_{i=1}^{r} \frac{\left\{2 k_{i}+1\right\} !}{\left\{k_{i}\right\} !\{1\}}\left(-f_{i} q\right)^{-f_{i} k_{i}\left(k_{3}+3\right) / 4}
\end{aligned}
$$

Hence

$$
J_{N}=\sum_{k_{1}, \ldots, k_{r}=0}^{\infty} \widetilde{C}\left(k_{1}, \ldots, k_{r}\right) \prod_{i=1}^{r}\left(-f_{i} q\right)^{-f_{i} k_{i}\left(k_{3}+3\right) / 4} .
$$

On the other hand, if $q^{1 / 4}$ is a root of unity of order $4 d$, then the quantum invariant $Z_{N}(q)$ is given by (see eg $[\mathrm{KM}]$ ) 


$$
Z_{N}(q)=\frac{\sum_{n_{i}=1}^{4 d} J_{\mathcal{L}^{(0)}}\left(n_{1}, \ldots, n_{r}\right) \prod_{i=1}^{r}\left[n_{i}\right] q^{\frac{f_{i}}{4}\left(n_{i}^{2}-1\right)}}{\prod_{i=1}^{r}\left(\sum_{j=1}^{4 d}\left[n_{i}\right]^{2} q^{\frac{f_{i}}{4}\left(n_{i}^{2}-1\right)}\right)}
$$

which, by (14) and Corollary 2.4 below, is equal to the right hand side of (15).

2.5. Laplace transform. Suppose $q^{1 / 4}$ is a primitive root of unity of order $4 d$. For $f= \pm 1$, Let $\gamma_{f}$ be the Gauss sum

$$
\gamma_{f}:=\sum_{k=1}^{4 d} q^{\frac{f}{4}\left(k^{2}-1\right)}
$$

The exact value of $\gamma_{f}$ can be calculated, see eg. [KM], but for us it is only important that $\gamma_{f} \neq 0$.

We define the Laplace transform $\mathbf{L}_{f ; n}$ as follows

$$
\mathbf{L}_{f ; n}\left(q^{n a+b}\right):=q^{b-f a^{2}} .
$$

Lemma 2.2. For any integers $a, b$ we have

$$
\sum_{n=1}^{4 d} q^{\frac{f}{4}\left(n^{2}-1\right)} q^{b+n a}=\gamma_{f} \mathbf{L}_{f ; n}\left(q^{b+n a}\right) .
$$

Proof. We have

$$
q^{\frac{f}{4}\left(n^{2}-1\right)} q^{b+n a}=q^{\frac{f}{4}\left((n+2 a)^{2}-1\right)} q^{b-f a^{2}} .
$$

Summing up $n$ from 1 to $4 d$ we get the result.

The following lemma, in another notation, was Lemma 2.2 of $\mathrm{BBL}$ :

Lemma 2.3. One has

$$
\mathbf{L}_{f, n}([n] C(n, k) /\{1\})=2\left(q^{-f}-1\right)(-f q)^{-f k(k+3) / 4} \frac{\{2 k+1\} !}{\{k\} !\{1\}} .
$$

Corollary 2.4. For $q^{1 / 4}$ a root of unity of order $4 d$, one has

$$
\frac{\sum_{n=1}^{4 d} q^{\frac{f}{4}\left(n^{2}-1\right)}[n] C(n, k) /\{1\}}{\sum_{n=1}^{4 d} q^{\frac{f}{4}\left(n^{2}-1\right)}[n]^{2}}=(-f q)^{-f k(k+3) / 4} \frac{\{2 k+1\} !}{\{k\} !\{1\}} .
$$

Remark 2.5. Despite the appearance of powers of $q^{1 / 4}$ in $\omega^{ \pm}, J_{N}$ contains integral powers of $q$. This follows from

$$
\begin{aligned}
q^{n(n+3) / 4} \frac{\{2 n+1\} !}{\{n\} !\{1\}} & =(-1)^{n} q^{-n(n+1) / 2} \frac{\{2 n+1\}_{-} !}{\{n\}_{-} !\{1\}_{-}} \\
(-1)^{n} q^{-n(n+3) / 4} \frac{\{2 n+1\} !}{\{n\} !\{1\}} & =q^{-n(n+2)} \frac{\{2 n+1\}_{-} !}{\{n\}_{-} !\{1\}_{-}}
\end{aligned}
$$

where the unbalanced quantum integers and factorials are defined by:

$$
\{n\}_{-}=1-q^{n}, \quad\{n\}_{-} !=\{1\}_{-} \ldots\{n\}_{-} .
$$

The next corollary gives a formula for $J_{N}$, where $N$ is obtained by \pm 1 surgery on a $\operatorname{knot} K$ in $S^{3}$ : 
Corollary 2.6. If $S_{K, \pm 1}^{3}$ denotes the result of \pm 1 surgery on a knot $K$ in $S^{3}$, then

$$
\begin{aligned}
J_{S_{K,-1}^{3}} & =\sum_{k=0}^{\infty} J_{K}\left(P_{k}^{\prime \prime}\right) q^{k(k+3) / 4} \frac{\{2 k+1\} !}{\{k\} !\{1\}} \\
& =\sum_{k=0}^{\infty} J_{K}\left(P_{k}^{\prime \prime}\right)(-1)^{k} q^{-k(k+1) / 2} \frac{\{2 k+1\}_{-} !}{\{k\}-!\{1\}_{-}} \\
J_{S_{K,+1}^{3}} & =\sum_{k=0}^{\infty} J_{K}\left(P_{k}^{\prime \prime}\right)(-1)^{k} q^{-k(k+3) / 4} \frac{\{2 k+1\} !}{\{k\} !\{1\}} \\
& =\sum_{k=0}^{\infty} J_{K}\left(P_{k}^{\prime \prime}\right) q^{-k(k+2)} \frac{\{2 k+1\}_{-} !}{\{k\}_{-} !\{1\}_{-}} .
\end{aligned}
$$

\section{A RELATIVE VERSiON}

Suppose $\mathcal{L}$ is an algebraically split $r$-component link in an integer homology 3 -sphere $N$, each component of which has framing 0 . It is known that there is an algebraically split framed link in $S^{3}$, which is the disjoint union of 2 sublinks $\mathcal{L}_{1}$ and $\mathcal{L}_{2}$, such that the framing of each component of $\mathcal{L}_{2}$ is \pm 1 , and that the surgery along $\mathcal{L}_{2}$ transforms $\left(S^{3}, \mathcal{L}_{1}\right)$ to $(N, \mathcal{L})$. Then $\mathcal{L}_{1}$ has $r$ components, each with framing 0 . Assume that $\mathcal{L}_{2}$ has $s$ components whose framings are $f_{1}, f_{2}, \ldots, f_{s}$. Let $\mathcal{L}^{(0)}$ be the link $\mathcal{L}_{1} \cup \mathcal{L}_{2}$ with all the framings switched to 0 .

Suppose $n_{1}, \ldots, n_{r}$ are positive integer. Let $Z_{N, \mathcal{L}}\left(n_{1}, \ldots, n_{r} ; \xi\right)$ be the WRT invariant of the pair $(N, \mathcal{L})$, when $\mathcal{L}$ is colored by the $U_{q}\left(\mathfrak{s l}_{2}\right)$-module $V_{n_{1}}, \ldots, V_{n_{r}}$, at the root $\xi$ of unity of order divisible by 4 . Our $\xi$ here (which is $q^{1 / 4}$ ) is equal to $t$ in [KM].

\subsection{WRT invariant $Z_{(N, \mathcal{L})}$ as element of $\widehat{\Lambda}$.}

Lemma 3.1. For positive integer $n_{1}, \ldots, n_{r}$, one has $J_{\mathcal{L}^{(0)}}\left(n_{1}, \ldots, n_{r}, \omega^{-f_{1}}, \ldots, \omega^{-f_{s}}\right)$ belongs to $\widehat{\Lambda}$.

Proof. Using (13) and a computation analogous to the one in the proof of Proposition 2.1 one has

$$
J_{\mathcal{L}^{(0)}}\left(n_{1}, \ldots, n_{r}, \omega^{-f_{1}}, \ldots, \omega^{-f_{s}}\right)=\sum_{0 \leq k_{i}<n_{i}}\left(\prod_{i=1}^{r} \frac{C\left(n_{i}, k_{i}\right)}{\{1\}}\right) D\left(k_{1}, \ldots, k_{i}\right)
$$

where

$$
D\left(k_{1}, \ldots, k_{r}\right)=\sum_{l_{1}, \ldots, l_{s}=0}^{\infty}\left(\prod_{i=1}^{s}\left(-f_{i} q\right)^{-f_{i} l_{i}\left(l_{i}+3\right) / 4}\right) J_{\mathcal{L}^{(0)}}\left(P_{k_{1}}^{\prime \prime}, \ldots, P_{k_{r}}^{\prime \prime}, P_{l_{1}}^{\prime}, \ldots, P_{l_{s}}^{\prime}\right) .
$$

Theorem 2 implies that

$$
J_{\mathcal{L}^{(0)}}\left(P_{k_{1}}^{\prime \prime}, \ldots, P_{k_{r}}^{\prime \prime}, P_{l_{1}}^{\prime}, \ldots, P_{l_{s}}^{\prime}\right) \in\left(\prod_{i=1}^{r} \frac{\left\{k_{i}\right\} !\{1\}}{\left\{2 k_{i}+1\right\} !}\right) \frac{\{2 m+1\} !}{\{m\} !\{1\}} \mathcal{R}
$$

where $m=\max \left\{k_{1}, \ldots, k_{r}, l_{1}, \ldots, l_{s}\right\}$. Using the easily verified identity

$$
\frac{C(n, k)}{\{1\}} \frac{\{k\} !\{1\}}{\{2 k+1\} !}=\left[\begin{array}{c}
n+k \\
2 k+1
\end{array}\right]\{k\} !
$$

we see that

$$
\prod_{i=1}^{r} \frac{C\left(n_{i}, k_{i}\right)}{\{1\}} J_{\mathcal{L}^{(0)}}\left(P_{k_{1}}^{\prime \prime}, \ldots, P_{k_{r}}^{\prime \prime}, P_{l_{1}}^{\prime}, \ldots, P_{l_{s}}^{\prime}\right) \in\left(\prod_{i=1}^{r}\left\{k_{i}\right\} !\right) \frac{\{2 m+1\} !}{\{m\} !\{1\}} \mathcal{R} .
$$

Hence for fixed $k_{1}, \ldots, k_{r}$, the term in the sum of the right hand side of (20) is in $\widehat{\Lambda}$. 
Recall that if $q^{1 / 4}$ is a root of unity of order $4 d$, then the WRT invariant is defined by

$$
Z_{(N, \mathcal{L})}\left(n_{1}, \ldots, n_{r} ; q\right)=\frac{\sum_{n_{i}=1}^{4 d} J_{\mathcal{L}^{(0)}}\left(n_{1}, \ldots, n_{r}\right) \prod_{i=1}^{r}\left[n_{i}\right] q^{\frac{f_{i}}{4}\left(n_{i}^{2}-1\right)}}{\prod_{i=1}^{r}\left(\sum_{j=1}^{4 d}\left[n_{i}\right]^{2} q^{\frac{f_{i}}{4}\left(n_{i}^{2}-1\right)}\right)} .
$$

Hence the proof of Proposition 2.1] can be easily generalized to the relative case, and one gets

Proposition 3.2. The evaluation of $J_{\mathcal{L}^{(0)}}\left(n_{1}, \ldots, n_{r}, \omega^{-f_{1}}, \ldots, \omega^{-f_{s}}\right)$ at a root $q^{1 / 4}=\xi$ of unity coincides with the quantum invariant $Z_{(N, \mathcal{L})}\left(n_{1}, \ldots, n_{r}\right)$ at that same root of unity.

Hence $J_{\mathcal{L}^{(0)}}\left(n_{1}, \ldots, n_{r}, \omega^{-f_{1}}, \ldots, \omega^{-f_{s}}\right)$ is an invariant of the link $\mathcal{L}$ in $N$, colored by $n_{1}, \ldots, n_{r}$.

Remark 3.3. Habiro's argument can directly show that $J_{\mathcal{L}^{(0)}}\left(n_{1}, \ldots, n_{r}, \omega^{-f_{1}}, \ldots, \omega^{-f_{s}}\right)$ is an invariant of the link $\mathcal{L}$ in $N$, colored by $n_{1}, \ldots, n_{r}$.

Let us denote $J_{\mathcal{L}^{(0)}}\left(n_{1}, \ldots, n_{r}, \omega^{-f_{1}}, \ldots, \omega^{-f_{s}}\right)$ by $J_{(N, \mathcal{L})}\left(n_{1}, \ldots, n_{r}\right)$, which is an element of $\widehat{\Lambda}$. We can consider $J_{(N, \mathcal{L})}$ as a function from $\mathbb{N}^{r}$ to $\widehat{\Lambda}$.

\section{4. $q$-HOLONOMICITY IN MANY VARIABLES}

Theorem 1 is a special case of Theorem 3 below, which follows from the fact that the quantum invariants can be built from elementary blocks that are $q$-holonomic, and the operations that patch the blocks together to give the colored Jones function preserve $q$-holonomicity. First we need the notion of $q$-holonomicity in many variables, introduced by Sabbah [C], generalizing Bernstein's notion of (usual) holonomicity [B1, B2].

4.1. $q$-holonomicity in many variables. Consider the operators $L_{i}$ and $M_{j}$ for $1 \leq i, j \leq r$ which act on functions $f$ from $\mathbb{N}^{r}$ to a $\mathbb{Z}\left[q^{ \pm 1 / 2}\right]$-module by

$$
\begin{aligned}
\left(M_{i} f\right)\left(n_{1}, \ldots, n_{r}\right) & =q^{n_{i} / 2} f\left(n_{1}, \ldots, n_{r}\right) \\
\left(L_{i} f\right)\left(n_{1}, \ldots, n_{r}\right) & =f\left(n_{1}, \ldots, n_{i-1}, n_{i}+1, n_{i+1}, \ldots, n_{r}\right) .
\end{aligned}
$$

It is easy to see that the following relations hold:

$$
\begin{aligned}
M_{i} M_{j} & =M_{j} M_{i} & L_{i} L_{j} & =L_{j} L_{i} \\
M_{i} L_{j} & =L_{j} M_{i} \text { for } i \neq j & & L_{i} M_{i}=q^{1 / 2} M_{i} L_{i}
\end{aligned}
$$

We define the $r$-dimensional quantum space $\mathcal{A}_{r}$ to be a noncommutative algebra with presentation

$$
\mathcal{A}_{r}=\frac{\mathbb{Z}\left[q^{ \pm 1 / 2}\right]\left\langle M_{1}, \ldots, M_{r}, L_{1}, \ldots, L_{r}\right\rangle}{\left(\operatorname{Rel}_{q}\right)} .
$$

For a function $f$ as above one can define the left ideal $\mathcal{I}_{f}$ in $\mathcal{A}_{r}$ by

$$
\mathcal{I}_{f}:=\left\{P \in \mathcal{A}_{r} \mid P f=0\right\} \text {. }
$$

If we want to determine a function $f$ by a finite list of initial conditions, it does not suffice to ensure that $f$ satisfies one nontrivial recursion relation if $r \geq 2$. The key notion that we need instead is $q$-holonomicity. Intuitively, $f$ is $q$-holonomic if it satisfies a maximally overdetermined system of linear difference equations with polynomial coefficients. The exact definition of holonomicity is through homological dimension, as follows.

Suppose $I$ is a left $\mathcal{A}_{r}$-module. Let $F_{m}$ be the sub-space of $\mathcal{A}_{r}$ spanned by polynomials in $M_{i}, L_{i}$ of total degree $\leq m$. Then the module $\mathcal{A}_{r} / I$ can be approximated by the sequence $F_{m} /\left(F_{m} \cap I\right), m=1,2, \ldots$ It turns out that, for $m>>1$, the dimension (over the fractional field $\left.\mathbb{Q}\left(q^{1 / 2}\right)\right)$ of $F_{m} /\left(F_{m} \cap I\right)$ is a polynomial in $m$ whose degree is called the homological dimension of $\mathcal{A}_{r} / I$ and is denoted by $d\left(\mathcal{A}_{r} / I\right)$.

Bernstein's famous inequality (proved by Sabbah in the $q$-case) states that the dimension of a non-0 module is $\geq r$, if the module has no monomial torsions, i.e., any non-trivial element of the module cannot be annihilated by a monomial in $M_{i}, L_{i}$. Note that the left $\mathcal{A}_{r}$-module $\mathcal{A}_{r} / \mathcal{I}_{f}$ does not have monomial torsion.

We say that a discrete function $f$ is $q$-holonomic if $d\left(\mathcal{A}_{r} / \mathcal{I}_{f}\right) \leq r$. Note that if $f$ is $q$-holonomic, then by Bernstein's inequality, either $\mathcal{A}_{r} / \mathcal{I}_{f}=0$ or $d\left(\mathcal{A}_{r} / \mathcal{I}_{f}\right)=r$. The former can happen only if $f=0$. 
4.2. Assembling $q$-holonomic functions. Here are some important operations that preserve $q$-holonomicity:

- Sums and products of $q$-holonomic functions are $q$-holonomic.

- Specializations and extensions of $q$-holonomic functions are $q$-holonomic. In other words, if $f\left(n_{1}, \ldots, n_{m}\right)$ is $q$-holonomic, the so are the functions $g\left(n_{2}, \ldots, n_{m}\right):=f\left(a, n_{2}, \ldots, n_{m}\right)$ and $h\left(n_{1}, \ldots, n_{m}, n_{m+1}\right):=$ $f\left(n_{1}, \ldots, n_{m}\right)$.

- Diagonals of $q$-holonomic functions are $q$-holonomic. In other words, if $f\left(n_{1}, \ldots, n_{m}\right)$ is $q$-holonomic, then so is the function

$$
g\left(n_{2}, \ldots, n_{m}\right):=f\left(n_{2}, n_{2}, n_{3}, \ldots, n_{m}\right) .
$$

- Linear substitution. If $f\left(n_{1}, \ldots, n_{m}\right)$ is $q$-holonomic, then so is the function, $g\left(n_{1}^{\prime}, \ldots, n_{m^{\prime}}^{\prime}\right)$, where each $n_{j}^{\prime}$ is a linear function of the $n_{i}$ 's.

- Multisums of $q$-holonomic functions are $q$-holonomic. In other words, if $f\left(n_{1}, \ldots, n_{m}\right)$ is $q$-holonomic, the so are the functions $g$ and $h$, defined by

$$
g\left(a, b, n_{2}, \ldots, n_{m}\right):=\sum_{n_{1}=a}^{b} f\left(n_{1}, n_{2}, \ldots, n_{m}\right), \quad h\left(a, n_{2}, \ldots, n_{m}\right):=\sum_{n_{1}=a}^{\infty} f\left(n_{1}, n_{2}, \ldots, n_{m}\right)
$$

(assuming that the latter sum is finite for each $a$ ).

For a user-friendly explanation of these facts and for many examples, see [Ze] and [PWZ].

4.3. Examples of $q$-holonomic functions. The following functions are $q$-holonomic:

$$
\begin{gathered}
n \rightarrow\{n\}, \quad n \rightarrow[n], \quad n \rightarrow[n] !:=\prod_{i=1}^{n}[i], \quad n \rightarrow\{n\} ! \\
(n, k) \rightarrow\{n\}_{k}:= \begin{cases}\prod_{i=1}^{k}\{n-i+1\}, & \text { if } k \geq 0 \\
0 & \text { if } k<0\end{cases} \\
(n, k) \rightarrow\left[\begin{array}{l}
n \\
k
\end{array}\right]:= \begin{cases}\frac{\{n\}_{k}}{\{k\}_{k}} & \text { if } k \geq 0 \\
0 & \text { if } k<0\end{cases}
\end{gathered}
$$

Also $q$-holonomic is the delta function $\delta_{n, k}$. In fact, we will encounter only sums, products, extensions, specializations, diagonals, and multisums of the above functions.

\section{4. $q$-holonomicity of quantum invariants.}

Theorem 3. For a 0-framed, algebraically split, $r$-component, oriented link $\mathcal{L}$ in an integer homology 3 sphere $N$, the function $J_{(N, \mathcal{L})}: \mathbb{Z}^{r} \rightarrow \widehat{\Lambda}$ is q-holonomic.

Proof. From GL we know that the function

$$
R(n, k):=(-1)^{n+1-k} \frac{\{1\}\{2 k\}}{\{n+1-k\} !\{n+1+k\} !}
$$

is $q$-holonomic, and that

$$
P_{n}^{\prime \prime}=\sum_{k=1}^{n+1} R(n, k) V_{k}
$$

By the result of [GL we know that $J_{\mathcal{L}^{(0)}}\left(k_{1}, \ldots, k_{r}, l_{1}, \ldots, l_{s}\right)$ is $q$-holonomic in all variables, hence $J_{\mathcal{L}^{(0)}}\left(P_{k_{1}}^{\prime \prime}, \ldots, P_{k_{r}}^{\prime \prime}, P_{l_{1}}^{\prime}, \ldots, P_{l_{s}}^{\prime}\right)$ is $q$-holonomic in all variables $k_{1}, \ldots, k_{r}, l_{1}, \ldots, l_{s}$. It follows from (21) that $D\left(k_{1}, \ldots, k_{r}\right)$ is $q$-holonomic, since $\left(-f_{1} q\right)^{-f_{1} k_{1}\left(k_{1}+3\right) / 4} \ldots\left(-f_{r} q\right)^{-f_{r} k_{r}\left(k_{r}+3\right) / 4}$ is $q$-holonomic in all variables. Then equation (20) shows that $J_{(N, \mathcal{L})}$ is $q$-holonomic. 


\section{REFERENCES}

[A] D. Bar-Natan, S. Garoufalidis, L. Rozansky and D. Thurston, The Aarhus integral of rational homology 3-spheres III: The relation with the Le-Murakami-Ohtsuki invariant, Selecta Math. 10 (2004) 305-324.

[BBL] A. Beliakova, C. Blanchet, and T. T. Q. Le, Laplace transform and universal sl(2) invariants, preprint 2005 math.QA/0509394

[B1] I.N. Bernstein, Modules over a ring of differential operators. An investigation of the fundamental solutions of equations with constant coefficients, Functional Analysis and its applications 5 1971 1-16, English translation: 89-101.

[B2] _ The analytic continuation of generalized functions with respect to a parameter, Functional Analysis and its applications 61972 26-40, English translation: 273-285.

[C] P. Cartier, Démonstration "automatique" d'identités et fonctions hypergéométriques (d'aprés D. Zeilberger), Séminaire Bourbaki, Vol. 1991/92. Astérisque No. 206 (1992), Exp. No. 746 41-91.

[CCGLS] D. Cooper, D, M. Culler, H. Gillet, D. Long and P. Shalen, Plane curves associated to character varieties of 3manifolds, Invent. Math. 118 (1994) 47-84.

[GL] S. Garoufalidis and TTQ. Le, The colored Jones function is q-holonomic Geometry and Topology, 9 (2005), 12531293.

[Ga] On the characteristic and deformation varieties of a knot, preprint 2003 math.GT/0306230 Proceedings of the CassonFest, Geometry and Topology Monographs 7 (2004) 291-309.

[Ha1] K. Habiro, On the quantum $\mathfrak{s l}_{2}$ invariants of knots and integral homology spheres Geom. Topol. Monogr. 4 (2002) $55-68$.

[Ha2] Habiro, Kazuo Cyclotomic completions of polynomial rings, Publ. Res. Inst. Math. Sci. 40 (2004) $1127-1146$.

[Ha3] , Bottom tangles and universal invariants, preprint 2005 math.GT/0505219

[Hi] K. Hikami, Difference equation of the colored Jones polynomial for torus knot, preprint 2004 math.GT/0403224

[Jan] J.C. Jantzen, Lecture on quantum groups, Graduate Studies in Mathematics, vol 6, AMS 1995.

[J] V. Jones, Hecke algebra representation of braid groups and link polynomials, Annals Math. 126 (1987) p. 335-388.

[Ka] L. Kauffman, State models and the Jones polynomial, Topology 26 (1987) 395-407.

$[\mathrm{KM}] \quad$ R. Kirby and P. Melvin, The 3-manifold invariants of Witten and Reshetikhin-Turaev for $\mathrm{sl}(2, C)$, Invent. Math. 105 (1991), 473-545.

[MM] P. Melvin and H. Morton, The coloured Jones function, Comm. Math. Phys. 169 (1995), 501-520.

[LMO] T.T.Q. Le, J. Murakami, T. Ohtsuki, A universal quantum invariant of 3-manifolds, Topology 37 (1998) 539-574.

[Le1] _ Integrality and symmetry of quantum link invariants, Duke Math. J., 102 (2000) 273-306.

[Le2] - The Colored Jones Polynomial and the A-Polynomial of Knots, preprint 2004 math.GT/0407521

[Le3] Strong Integrality of Quantum Invariants of 3-manifolds, preprint 2005 math.GT/0512433

[O] T. Ohtsuki, A study of knots, 3-manifolds, and their sets, Series on Knots and Everything, 29 World Scientific 2002.

[PWZ] M. Petkovšek, H.S. Wilf and D.Zeilberger, $A=B$, A.K. Peters, Ltd., Wellesley, MA 1996.

[PS] J. Przytycki and A. Sikora, Skein algebra of a group, Knot theory, 297-306, Banach Center Publ., 42, Polish Acad. Sci., Warsaw, 1998.

[RT] N. Reshetikhin, V. Turaev, Ribbon graphs and their invariants derived from quantum groups, Commun. Math. Phys. 127 (1990) 1-26.

[S] C. Sabbah, Systémes holonomes d'équations aux q-différences, in D-modules and microlocal geometry, Lisbon (1990) 125-147 de Gruyter, Berlin, 1993.

[Tu] V. Turaev, Quantum invariants of knots and 3-manifolds, de Gruyter Studies in Mathematics, vol. 18. Walter de Gruyter \& Co., Berlin, 1994.

[Ze] D. Zeilberger, A holonomic systems approach to special functions identities, J. Comput. Appl. Math. 32 (1990) 321-368.

[Za] D. Zagier, Vassiliev invariants and a strange identity related to the Dedekind eta-function, Topology 40 (2001) 945-960.

School of Mathematics, Georgia Institute of Technology, Atlanta, GA 30332-0160, USA, http://www.math.gatech .edu/ stavros

E-mail address: stavros@math.gatech.edu

School of Mathematics, Georgia Institute of Technology, Atlanta, GA 30332-0160, USA, http://www.math.gatech .edu/ letu

E-mail address: letu@math.gatech.edu 